\title{
Editorial: 2011 in Review
}

\author{
Fred R. Volkmar
}

Published online: 10 February 2012

(C) Springer Science+Business Media, LLC 2012

As has now been the case for several years, both the quality and quantity of research in autism has continued to increase dramatically. This past year, we instituted a policy of blinded reviews that has generally been found helpful by both authors and reviewers. We've also become more vigilant about the issue of duplicate publication and, sadly, have dealt with one instance of plagiarism (the first author was admonished, the paper rejected, and a letter went to the co-authors and the institution). During 2011, a record number of papers, reviews, and letters to the editor, 712 in total, were submitted. As in the past, the able staff both at Springer and my office and those of the associate editors have worked diligently to ensure that the papers conform to Journal guidelines before they are sent for review. As a practical matter, this means that most papers are returned quickly to the authors for revision even before going out for peer review. Whenever possible, we attempt to be expeditious with reviews-the turnaround time averaged about 8 weeks (with a few reviews being much faster and some being much slower). One of the problems that we, and other journals, face is 'reviewer fatigue'. We have taken several steps to deal with the increase in submissions. The associate editors and I have now begun, occasionally to reject papers (about 5\%) that we believe simply have little or no chance of making it through the peer review process.

As noted previously, we have stopped accepting case reports except as letters to the editor (these take a disproportionate burden of time when submitted as regular papers and create special problems in terms of duplicate publication). We are enlarging our editorial board, and I'm

F. R. Volkmar $(\square)$

Yale Child Study Center, New Haven, CT, USA

e-mail: fred.vokmar@yale.edu pleased to welcome both the new members of the board, as we bid farewell to those rotating off. We are pleased to welcome two new associate editors to the Journal.

Dr. Joshua Diehl from the University of Rochester will replace Dr. Rhea Paul as she steps down from her role of associate editor later this year. Dr. Diehl's work has focused on improving and understanding social-communication problems in autism. His interests in such areas as adaptive technology bring another important strength to the board. We are also pleased to welcome Dr. Jeremy Veenstra-VanderWeele from Vanderbilt University as a new associate editor. Dr. Veenstra-VanderWeele's work has focused on animal models and the neurobiology of autism and brings another important perspective to the Journal. Finally, Dr. Brian Reichow, a special educator and expert in evidence-based treatments and practice, is joining us as Book Review Editor.

Although $J A D D$ is now published every month, the increasing number of submissions means that the rejection rate will continue to increase. Most papers (about 80\%) are now revised to ensure that they conform to Journal style even before they enter the review process. Of the papers submitted in 2011 in which an editorial decision was made, the acceptance rate was approximately $38 \%$ compared to $45 \%$ in 2010; this does not include the small number of papers rejected without review. As always, the diligent efforts of the Editorial Board ensure that the leading scientific papers are selected for publication in $J A D D$ each year.

As always I'm deeply grateful to the Associate Editors: Nancy Minshew, M.D., Sandra Harris, Ph.D., Christopher McDougle, M.D., Rhea Paul, Ph.D. and Tony Charman, Ph.D. who have so generously and diligently supported the work of the Journal even in the face of the growing number of submissions. I'm also grateful to Lori Klein and Emily 
Hau in the Journal office who work closely with authors to insure that papers are of high quality - at least in terms of their format-even before they enter the peer review process. We also thank Judy Jones at Springer and the individuals so involved in production of the JournalGarth Haller and David Seidenfeld. This year we bid farewell to the following members who have been on the board: Sander Begeer, VU University of Amsterdam, Sven Bolte, Karolinska Institutet, Ira L. Cohen, NYS Institute for Basic Research in Developmental Disabilities, Peter Doehring, Delaware Autism Program, Tina Dyches, Brigham Young University, Hiroshi Kurita, University of Tokyo Faculty of Medicine. We also welcome the following new members of the board: Jarrett Barnhill, University of North Carolina School of Medicine, Catherine Barthélémy, University Hospital of Tours, Dorit Ben Shalom, University of the Negev, Vicki

Abadeh, Heidi

Abdelmeguid, Nabila

Abi-Dargham, Anissa

Abrams, Mark

Abu-Dahab, Sana

Achenbach, Thomas

Acosta, Maria

Adamek, Lauren

Adams, Catherine

Adams, Denise

Adams, Lynn

Adams, Nena

Adnopoz, Jean

Agnew, John

Aguiar, Sandra Maria Herondina

Coelho

Ahlem, Belhadj

Ahlert, Noelle

Ahmadi, Ahmad

Ahmed, Deka

Aitken, Kenneth

Akechi, Hironori

Akinci, Arsen

Akoury Dirani, Leyla

Al Anbar, Nebal

Albano, Anne

Alcántara, Jose

Alderson-Day, Ben

Aldred, Catherine

Aldridge, Fiona

Alexander, A.

Alexander, Lisa

Al-Farsi, Yahya

Allam, Hemat

Allen, Daniel
Allen, David

Allen, Rory

Allison, Carrie

Allman, M.J.

Alsayed, Eman

Altgassen, Mareike

Altschuler, Eric

Aman, Michael

Amaral, David

Ames, Catherine

Amin, Sanjiv

Amorim, Letícia

Anagnostou, Evdokia

Ananthan, Lata

Anckarsater, Henrik

Anderson, George

Anjum, Saliha

Annaz, D.

Antshel, Kevin

Apicella, Fabio

Aras, Sahbal

Araujo, Eva

Arciuli, Joanne

Arnold, Jennifer

Arnold, L. Eugene

Arora, Aarti

Asada, Kosuke

Åsberg, Jakob

Ashtari, Manzar

Ashwood, Paul

Assouline, Susan

Atkinson, Anthony

Atladóttir, Hjördís

Austin, Sarita

Axe, J.B.
Bitsika, Bonds University School of Social Sciences, Stephen M. Kanne, Baylor College of Medicine, Steve Kroupa University of North Carolina, Eli Lebowitz, Yale University School of Medicine, Donald P. Oswald, Virginia Commonwealth University, Bertram O. Ploog, College of Staten Island, Johannes Rojahn, George Mason University, Frederick Shic, Yale University School of Medicine, Eileen Nicole Simon, MHM Services, Bridgewater State Hospital, Alison Singer, Autism Science Foundation, David M. Williams, Durham University, and Virginia C.N. Wong, The University of Hong Kong.

At the conclusion of this editorial we provide a list of all those individuals who have contributed one or more reviews to the Journal this year. Although far from perfect peer review is the best way we have, as a field, in insuring the quality of our scholarly product. We thank all these individuals for their hard work and dedication.
Aylward, Elizabeth
Azeem, Muhammad
Bachmann, Christian
Back, Elisa
Baghdadli, Amaria
Baglio, Christopher
Baharav, Eva
Bahrick, Lorraine
Bailey, Karyn
Baird, Gillian
Bajaj, Shailesh
Bakare, Muideen
Baker, Dana Lee
Baker, Jason
Baker, Sara
Bakken, Trine Lise
Baldassii, Stefano
Ballan, Michelle
Banach, Ryan
Bandeira de Lima, Cláudia
Baptista, Patricia
Baranek, Grace
Barbaro, Josephine
Barbosa-Gonçalves, Adriana
Barcala, Alejandra
Barker, E.T.
Barnes, Gregory
Barnes, Jennifer
Barnes, Kelly
Barneveld, Petra
Barnhill, Gena
Barnhill, L. Jarrett
Baron Cohen, Simon
Barretto, A.
Barry, Tammy 
Barthelemy, Catherine

Bartley, Jane

Barton, Erin

Barton, Marianne

Barwood, C.

Battaglia, Agatino

Batu, Sema

Baudino, Lori

Bauer, Nerissa

Bauminger-Zviely, Nirit

Bavin, Edith

Bayat, Mojdeh

Bayliss, Andrew

Bayou, Nadia

Beall, Paula

Bear, M.F.

Beauchamp, M.H.

Beaumont, Renae

Beauvais, Jessica

Bebko, James

Beckwith, Malia

Begeer, Sander

Behrmann, Marlene

Beidel, Deborah

Bellini, Scott

Bellis, Terry

Belmonte, Matthew

Benites, Fabiana

Ben-Itzchak, Esther

Bennett, Emily

Bennett, Marc

Bennetto, Loisa

Ben-Sasson, Ayelet

Ben-Shalom, Dorit

Benson, Betsey

Benson, Paul R.

Benson, Sven

Bentley, Mary Jane

Berger, J.T.

Berger, M.

Bergquist, Thomas

Berkowitz, Steven

Berlin, Kristoffer

Bernabei, Paola

Bernal, Pilar

Berney, Thomas

Bernier, Raphael

Bernstein Ratner, Nan

Berry, David

Berry-Kravis, Elizabeth

Berthoz, Sylvie

Bertone, Armando

Best, Catherine
Best, Karin

Betz, Alison

Beuker, Karin

Beversdorf, David

Bhagwagar, Zubin

Bhat, Anjana

Bhatara, Anjali

Bhaumik, Sabayasachi

Bigda-Peyton, Joseph

Bigler, Erin

Billard, Aude

Bird, Geoffrey

Birdgemohan, Carolyn

Biscaldi, Monica

Bishop, Dorothy

Bishop, Somer

Bitsika, Vicki

Blacher, Jan

Black, David

Blair, Kwang-Sun

Blakeley-Smith, Audrey

Bland-Stewart, Linda

Blankenship, Kelly

Blann, Lauren

Blatt, Gene

Błeszyński, Jacek

Blijd-Hoogewys, Els

Bloch, Michael

Bloom, Sarah

Boddaert, Nathalie

Bodfish, James W.

Bodfish, Jim

Bogte, Hans

Bolduc, Francois

Bolman, William

Bolte, Sven

Bolton, Patrick

Bonneh, Yoram

Bookheimer, Susan

Booth, Leah

Booth, Rhonda

Bopp, Karen

Boraston, Zillah

Borham, Sharon

Borrego, Joaquin

Borrero, John

Bosl, William

Boso, Marianna

Bostrom, Petra

Bottema-Beutel, Kristen

Botting, Nicola

Boucher, Jill

Bouder, James
Boudreau, Donna

Boulet, Sheree

Bourgeois, James

Boutros, Nashaat

Bowker, Anne

Bowler, Dermot M.

Bowyer-Crane, Claudine

Boyd, Brian

Brackett, Marc

Bradley, Elspeth

Brady, Nancy

Bramham, Jessica

Bregman, Joel

Brian, Jessica A.

Brickhouse, T.H.

Brimacombe, Michael

Brinton, Bonnie

Brock, Jon

Brock, Matthew

Brodkin, Edward

Brookman-Frazee, Lauren

Brosnan, Mark

Brown, Alan

Brown, Alec

Brown, Amy

Brown, Benjamin

Brown, Caroline

Brown, Heather

Brown, John

Brown, Ted

Brownell, Kelly

Bruck, Maggie

Bruckner, Cordelia

Brugha, Traolach

Bruining, $\mathrm{H}$.

Bruni, Oliviero

Bryson, Susan

Buckingham, Mallory

Buckley, Ashura

Bühler, Eva

Buitelaar, Jan K.

Buizer-Voskamp, Jacobine

Bundy, Myra Beth

Burack, Jacob A.

Burkhart, Carolyn

Burnette, Courtney

Burrell, Teresa

Burrows, Kristen

Butler, Merlin

Butter, Eric

Buxbaum, Joseph

Buzanko, Caroline

Byiers, Breanne 
Cacace, Anthony

Calculator, Stephen

Calhoun, Susan

Callahan, Kevin

Camarata, Stephen

Campbell, Daniel

Campbell, Jonathan

Campbell, Martha Karen

Campbell, Ruth

Campbell, Thomas

Cannella-Malone, Helen

Caplan, Scott

Cappadocia, Mary Catherine

Carbone, Paul

Cardon, Teresa

Carlezon, Bill

Carlson, Derek

Carney-Doebbling, Caroline

Caronna, Elizabeth

Carothers, Douglas

Carpenter, Patricia A.

Carroll, Aaron

Carter, Alice

Carter, Susan

Cartwright-Hatton, Sam

Carver, Leslie

Casanova, Manuel

Cascio, Carissa

Cascio, Carissa

Case-Smith, Jane

Casey, Betty

Cashin, A.

Cashion, Larry

Castellanos, Francisco Xavier

Caterino, Linda

Cath, Danielle

Cauraugh, James

Cavanagh, Paul

Cavazos, Homero

Cederlund, Mats

Cermak, Sharon

Chalfant, Anne

Chan, Agnes

Chan, Jeffrey

Chang, Chih-Hui

Chang, Hsueh-Ling

Chang, Joseph

Chapman, Leigh

Charles, J.

Charman, Tony

Chawarska, Katarzyna

Cheak-Zamora, Nancy

Cheng, Yawei
Cheslack-Postava, Keely

Cheung, Sau Wai

Chiang, Hsu-Min

Childress, Deb

Chitiyo, Morgan

Chotirmall, Sanjay

Chou, Pesus

Christ, Shawn

Christensen, Lisa

Christodoulou, Manolis

Christopher, Sheila

Chugani, Diane

Chumney, Frances

Chung, Kyung Mee

Chung, Moo

Ciccarelli, Mary

Cicchetti, Domenic

Cirrin, F.

Cjarlop-Christy, Marjorie

Clapp, D. Wade

Clare, Isabel

Clark, T.F.

Clarke, Juanne

Classen, John

Cleland, Joanne

Clifford, Sally

Clifford, Tess

Clifton, Yeaton

Coates, $\mathrm{H}$.

Coben, Robert

Cobigo, Virginie

Cody-Hazlett, Heather

Coffey, M. Justin

Cohen, Brett

Cohen, Ira

Cohen, Phyllis

Colle, Livia

Colombi, Costanza

Colvert, Emma

Connor, Dan

Connors, Susan

Constantino, John

Cook, Jr., Edwin

Coolican, Jamesie

Coonrod, Elaine

Cooper, S.A.

Corbett, Blythe

Cordeiro, Lisa

Cormier, Damien

Cornetta, Kenneth

Correia, Natália

Costantini, Marcello

Cotugno, Albert
Coughlin, Michael

Coury, Daniel

Couture, Melanie

Cowie, Dorothy

Cox, David

Cox, Neill

Crais, Elizabeth

Cram, Alexandra L.

Crane, Laura

Crasta, Jewel

Crawford, Susan

Crawley, Jacqueline

Creedon, Margaret

Crespi, Bernard

Crewther, David

Croen, Lisa

Cronin, Pegeen

Crooke, Pamela

Crowley, Michael

Crozier, Shannon

Cuccaro, Michael

Curran, Laura

Curtin, Carol

Cuvo, Anthony

Dadds, M.R.

Dager, Stephen

Dahlgren Sandberg, Annika

Dale, Emily

Dale, Naomi

Dale, Philip

Dalton, Kim

Daniels, Amy

Danovitch, Judith

Dapretto, Mirella

Dautenhahn, Kerstin

David, Nicole

Davidson, Joyce

Davidson, Richard

Davies, Patricia

Davis, Aileen

Davis, Greg

Davis, Megan

Davis, Patricia

Davis, Tonya

Dawson, Geraldine

de Bildt, Annelies

de Bruin, Esther

de Jong, Marianne

de jonge, M.V.

de la Cruz, Berenice

de Los Reyes, Emily

de Nijs, Pieter

de Paula, Cristiane 
de Pauw, Sarah

de Vincent, Carla

de Vries, Annelou

de Vries, Marieke

Dealberto, Marie-José

Dean, Kim

Decety, J.

Delafield-Butt, Jonathan

Delaney, Mary Beth

Dempsey, Allison

D'Entremont, Barbara

Deruelle, Christine

DeSoto, M.C.

Dessai, Rashmi

Deth, Richard

Deutsch, Stephen

DeVivo, Darryl

Dichter, Gabriel

Dickie, Virginia

Didden, Robert

Diehl, Joshua

Dietz, Claudine

DiGuiseppi, Carolyn

Dille, Lisa

Dillenburger, Karola

Dingfelder, Hilary

Dion, Eric

Dissanayake, Cheryl

Djukic, Aleksandra

Dobkins, Karen

Dodds, Linda

Doehring, Peter

Dogra, Vikas

Doherty-Sneddon, Gwyneth

Doken, Ibrahim

Dolyniuk, Chrystina Anna

Donkers, Franc

Donnay, Albert

Dormans, John

Dowdy, E.

Downs, Stephen

Dowrick, Peter W.

Doyle, Carolyn

Drahota, Amy

Drake, Daniel

Drake, Jennifer

Drazin, Doniel

Dritschel, Barbara

Dubey, Indu

Duijkers, Judith

Duke, Jodi

Dunn, David

Dunn, Winnie
Durkin, Kevin

Durkin, Maureen

Duvignau, Karine

Dworzynski, Katharina

Dyck, Murray

Dykens, Elisabeth M

Eack, Shaun

Eagle, Rita

Eaton, William

Eaves, Linda C.

Eaves, Ronald C.

Eckart, Gina

Edgin, Jamie

Ehrenreich-May, Jill

Eigsti, Inge-Marie

Einspieler, Christa

Eisenberg, Nancy

Ek, Ulla

Ekas, Naomi

El-Ansary, Afaf

Eldevik, Sigmund

El-Fishawy, Paul

Elias, Ellen

Ellis Weismer, Susan

Elsabbagh, Mayada

Endo, Taro

Engels, Rutger C.M.E.

Enticott, Peter

Eren, Ruth Irene

Erickson, Craig

Eriksen, Mette Elmose

Erjavec, M.

Ernst, Monique

Esbensen, Anna

Esch, Barb

Esgin, Esad

Estes, Annette

Evans, Beverley

Evans, David

Evans, Julia

Evans-Smith, Bernadette

Fabricius, Thomas

Fahim, Donia

Faja, Susan

Falck-Ytter, Terje

Fallin, M.

Falter, Christine

Farah, Huda

Farley, Megan

Farmer, Cristan

Farrar, M.J.

Farzin, Faraz

Fatemi, S.
Faust, Miriam

Fauzan, Norsiah

Fawaz, Walid

Feeley, Kathleen

Fees, Bronwyn

Fein, Deborah

Feinstein, Carl

Felce, David

Fellner, Christian

Fernandez, Thomas

Fernell, Elisabeth

Fienup, Daniel

Filipek, Pauline

Findling, Robert

Fine, Jodene

Finucane, Brenda

Fiorio, M.

Fisher, Julia

Fitzgerald, Michael

Flachsbart, Celeste

Flapper, Boudien

Fletcher-Watson, Sue

Fleury, Amanda

Flint, Jonathan

Fodstad, Jill

Foley Nicpon, Megan

Folstein, Susan

Fombonne, Eric

Fong, Chin-To

Forsyth, Kirsty

Forti, Sara

Foss-Feig, Jennifer

Fox, Peter

Fox, Robert

Foxx, R.M.

Frankel, Frederick

Franklin, Martin

Franks, Robert

Freedman, Brian

Freeth, Megan

Freitag, Christine

Friedman, M.

Friesen, Chris

Frith, Uta

Fritsch, Amy

Fuentes Biggi, Joaquin

Fujii, Yutaka

Funabiki, Yasuko

Furman, Joseph

Gabis, Lidia

Gabriels, Robin

Gadow, Kenneth

Gage, Nicole M. 
Gahr, Maximilian

Gaigg, Sebastian

Gaitonde, Shital

Gal, Gilad

Galanopoulou, Aristea

Galasso, Cinzia

Galéra, Cédric

Gallese, Vittorio

Ganos, Christos

Gantman, Alexander

Ganz, Jennifer

Ganz, Michael

Garant, Amanda

García-Primo, Patricia

Gargaro, Belinda

Gargus, John

Gast, David

Gastgeb, Holly

Gathercole, Susan

Gauthier, Isabel

Gebhart, A.L.

Geller, Daniel

Georgiades, Stelios

Gepner, Bruno

Geraghty, Maureen

Gerhardt, Peter

Gerig, Guido

Germain-Lee, Emily

Gernsbacher, Morton

Geschwind, Daniel

Getchell, Nancy

Geurts, Hilde

Ghanizadeh, Ahmad

Ghaziuddin, Mohammad

Giannoulis, Katingo

Giardino, Daniela

Giarelli, Ellen

Gibbon, Fiona

Gibson, Jenny

Gilbert, Kimberly

Giles, Howard

Gillberg, Christopher

Gillott, Alinda

Ginsburg, Golda

Giovingo, Lauren

Girgis, Ragy

Glanzman, Marianne

Glaser, Sarah

Glazebrook, Cheryl

Glenwright, Melanie

Glickman, G.

Gliga, Teodora

Gogtay, Nitin
Goh, Tze Jui

Goin-Kochel, Robin

Golan, Ofer

Goldberg, Melissa

Goldman, Suzanne

Goldman, Sylvie

Goldsmith, Tina

Goldstein, Gerald

Goldstein, Howard

Goldstein, Sam

Golnik, Allison

Golterman, Linda

Gomes, Erissandra

Gomot, Marie

Goodman, Sabrina

Goodwin, Matthew

Gosse, Carolyn

Gotham, Katherine

Gothelf, Doron

Goudreau, Diane

Gowen, Emma

Goyette-Ewing, Michele Anne

Granpeesheh, Doreen

Grant, Cathy

Gray, Kylie

Greaves-Lord, Kirstin

Green, Gina

Green, Jonathan

Green, Shulamite

Green, Vanessa

Greenaway, Rebecca

Greenbaum, Rosalie

Greenough, William

Gregg, Jeffrey

Greimal, Ellen

Grether, Judith

Grice, Dorothy

Griffith, Elizabeth

Grigorenko, Elena

Grindle, Corinna

Gringras, Paul

Grinker, Roy

Grinter, Emma

Grodberg, David

Groen, Wouter

Gropman, Andrea

Grossman, Ruth

Grynszpan, Ouriel

Grzadzinski, Rebecca

Guardino, Caroline

Gunlogson, Christine

Gunnarsson, Ronny

Gupta, Abha
Gutstein, Steven

Guttmann-Steinmetz, Sarit

Haber, Mason

Hagberg, Bibbi

Hagerman, Paul

Hagerman, Randi

Halayem, soumeyya

Hall, Geoffrey

Hall, Heather R.

Hall, Kathleen

Hall, Scott S.

Halladay, Alycia

Hallerbäck, Maria

Hallett, Victoria

Hallmayer, Joachim

Haltigan, John

Hamilton, Antonia

Hampel, Petra

Handen, Benjamin L.

Handleman, Jan

Hannigen, Sarah

Hansen, Robin

Hansen, Robin

Hansford, Amy

Hao, Grace

Happe, Francesca

Harbin, Ginger

Hardan, Antonio

Hare, Dougal

Harmony, Max

Harrington, John W.

Harris, James C.

Harris, Jennifer

Harris, Jill

Harris, Jonathan

Harris, Sandra

Hart, Lesley

Hartley, Sigan

Haskins, Barbara

Hatton, Deborah

Havasi, Catherine

Hays, Marilyn

Healy, Olive

Heaton, Pamela

Hedley, Darren

Heidmann, Saylor

Heiman, Tali

Heimann, Mikael

Hellings, Jessica

Henderson, Heather

Hendren, Robert

Hepburn, Susan

Herba, Catherine 
Herbert, Martha

Herrero, $\mathrm{P}$.

Hershler, Orit

Hertle, Richard

Hertz-Picciotto, Irva

Hess, Kristen

Hessl, David

Hetrick, William

Hettinger, Joe

Hewitt, Lynn

Hilibrand, Deborah

Hill, David

Hill, Doris

Hill, Elisabeth

Hillier, Ashleigh

Hilton, Claudia

Hingtgen, Cynthia

Hinton, Veronica

Hippler, Kathrin

Hirschfeld, Lawrence

Ho, Helena

Hobson, Jessica

Hobson, Peter

Hoekstra, Rosa

Hofvander, Bjorn

Hogsbro, Kjeld

Hollander, Eric

Holm, Margo

Holmqvist, Mona

Holsen, Laura

Holtmann, Martin

Honey, Emma

Honigfeld, Lisa

Hooper, Stephen

Horlin, Chiara

Hornig, Mady

Horowitz, L.M.

Howard, Janice

Howlin, Patricia

Hrdlicka, Michal

Hsiao, Elaine

Hsiao, Janet

Hsu, Ching-fen

Huang, Henry

Hudenko, William

Hudry, Kristelle

Hudson, Matthew

Hudziak, James

Hughes, Claire

Hume, Kara

Hummel-Rossi, Barbara

Hummer, Tom

Humphrey, Neil
Hurst, Ruth

Hurwitz, Sarah

Hutchins, Gary

Hutchins, Tiffany

Hvidtjørn, Dorte

Hwang, Yoon-Suk

Hyman, Susan

Iacoboni, Marco

Iarocci, Grace

Imaizumi, Satoshi

Ingersoll, Brooke

Ingvarsson, Einar

Innis, Robert

Irani, F.

Irwin, Julia

Isenhower, Robert

Iverson, Jana

Jack, Allison

Jacobs, Diane

Jahromi, Laudan

Jakobson, Lorna

James, Jill

Jan, James

Jarrold, Christopher

Jarvin, Linda

Järvinen-Pasley, Anna

Jarvis, Patricia

Jarvis, Steve

Jellema, Tjeerd

Jelliffe-Pawlowski, Laura

Jemel, Boutheina

Jewell, Jeremy

Ji, Na Young

Johnson, Beth

Johnson, Connie

Johnson, Sam

Johnson, Valerie

Johnson, Velisa

Johnston, Kate

Jones, Alice

Jones, C.R.

Jones, Dennis

Jones, Erin

Jones, Garland

Joosten, Annette

Jordan, C.J.

Jordan, Iain

Joseph, Robert M.

Joshi, Gagan

Jou, Roger

Jukes, Matthew

Kagohara, Debora

Kaipa, Ramesh
Kaiser, Ann P.

Kaiser, Martha

Kaland, Nils

Kalbfleisch, Layne

Kamp-Becker, Inge

Kandalaft, Michelle

Kaniel, Shlom

Kanne, Stephen

Karanth, Prathibha

Kasari, Connie

Kates, Wendy

Kato, Shiho

Kaufman, Alan

Kaur, Archana

Kaur, Mandeep

Kawa, Rafal

Kawakubo, Yuki

Kay-Raining Bird, Elizabeth

Keck, Julie

Keehn, Brandon

Keen, Deb

Keller, Timothy

Kelly, A.B.

Kelsen, Brent

Kemner, Chantal

Kendall, Philip

Kennedy, Daniel

Kenworthy, Lauren

Kern, Janet

Kern, Petra

Khalfa, S.

Kidd, Sharon

Kiesel, Andrea

Kikuchi, Yukiko

Kim, Jinah

Kim, Jin-Kyung

Kim, Soo-Jeong

Kim, Young-Shin

Kimhi, Yael

King, Bryan

King, Robert

Kinney, D.K.

Kirchner, Jennifer

Klauk, S.M.

Klee, Thomas

Kleinhans, Natalia

Klein-Tasman, Bonita (Bonnie)

Kliemann, Dorit

Klin, Ami

Klinger, Laura

Klintwall, Lars

Knapp, Julie

Knickmeyer, Rebecca 
Knollmann, Martin

Knott, Fiona

Kodak, Tiffany

Koegel, Lynn

Koegel, Robert

Koelch, Michael

Koenig, Kathy

Kogan, C.S.

Kogan, Michael

Kohls, Gregor

Kokina, Anastasia

Kolassa, Iris

Kolevzon, Alexander

Koneru, A.

Konstantareas, Marianthi

Konstantynowicz, Jerzy

Kopycka-Kedzierawski, Dorota

Korkmaz, B.

Koshino, Hideya

Kourkoulou, Anastasia

Kovshoff, Hanna

Koyama, Tomok

Kozima, H.

Kraemer, Bernd

Krebs, Julia

Kroeger, Anne

Kroeger, Kimberly

Kronenberger, William

Kroupa, Steve

Krug, David

Kuder, S. Jay

Kuhn, Gustav

Kunda, Maithilee

Kurita, Hiroshi

Kuschner, Emily

Kuusikko, Sanna

Kuzmanovic, Bojana

Kylliainen, Anneli

Lachiewicz, Ave M.

Lagges, Ann

Lahiri, Debomoy

Lai, M.C.

Lainé, France

Lainhart, Janet

Lajiness-O’Neill, Renee

Lam, Kristen

Lambrechts, Greet

Lamothe, Julie

Lampi, Katja

Landa, Rebecca

Landi, Nicole

Landry, Susan

Landsberger, Sarah
Lane, Alison

LaRue, Robert

Lasgaard, Mathias

Laud, Rinita

Laugeson, Elizabeth

Lauritsen, Marlene

Laushey, Kelle

Law Smith, Miriam

Lawlor, Debbie

Lawrence, Diane

Lawton, Kathryn

Layton, Thomas L.

Le Couteur, Ann

Leaf, Justin

Leaf, Ronald

LeBlanc, Linda

Lebowitz, Eli

Lecavalier, Luc

Lechner, Helga

Leckman, James

Lee, Alice

Lee, Brian

Lee, Douglas

Lee, Li-Ching

Lee, Ronald

Lee, Sue

Leekam, Susan

LeGoff, Daniel

Leighton, Jane

Lennen, Daniel

Lenroot, R.K.

Leonard, Hayley

Leonard, Helen

Lerner, Matthew

Leslie, Alan M.

Leslie, Laurel

Lesser, Ronald

Leu, Roberta

Levine, Todd

Levitt, Pat

Levy, Kenneth

Levy, Susan

Lewandowski, Thomas

Lewis, Charlie

Lewis, Mark

Lewis, Pamela

Leyfer, Ovsanna

Li, Ning

Liamputtong, Pranee

Libertus, Klaus

Lieberman, Rebecca

Lifter, Karin

Light, Janice
Limperopoulos, Catherine

Lincoln, Alan

Lind, Sophie

Lindell, A.K.

Linden, Mark

Lindsay, Ronald

Ling, J.

Liptak, Gregory

Liss, Miriam

Liu, Ting

Lloyd, Meghann

Locke, Jill

Lockner, Donna

Loddo, Silvio

Loftin, Rachel

Logemann, H.

Lombardo, Michael

London, Eric

Longo, M.R.

Loo, Cheen

Lopata, Christopher

Lopez, Beatriz

Lopez-Cohen, Gwendolyn

Lopez-Wagner, Muriel

Lord, Catherine

Losh, Molly

Loth, Eva

Lotspeich, Linda

Lotstein, Debra

Loucas, Tom

Loukusa, Soile

Love, Steven

Loveland, Katherine

Lozano, Milagros

Luchansky, Craig A.

Lucyshyn, Joseph

Lugnegard, Tove

Luiselli, James

Luke, Lydia

Luna, Beatriz

Lunsky, Yona

Luthy, Beth

Luts, Jan

Luyster, Rhiannon

Lyons, Viktoria

M'rad, Ridha

Macari, Suzanne

Maciver, Donald

Mack, Hilary

MacKay, Tommy

Maclean, Bill

Macomber, Donna

Maenner, Matthew 
Maes, Joseph

Magiati, Iliana

Magliaro, Fernanda

Maglione, Margaret

Magnusson, Jane

Magyer, Caroline

Maimburg, Rikke

Maister, Lara

Male, Ian

Malison, Robert

Maljaars, Jarymke

Malmberg, Debra Berry

Malone, Richard

Malow, Beth

Mancil, Gregory

Mandell, David

Mandy, William

Mangeot, Shanley

Manjiviona, Janine

Marans, Wendy

Maras, Katie

Marcus, Lee

Marinova-Todd, Stefka

Markram, Kamila

Marks, Lawrence

Marschik, Peter

Martens, Marilee

Martin, Andrés

Martineau, Joelle

Martinez-Pedraza, Frances

Martins, Megan

Masino, Susan

Mateljevic, Natasa

Matlock, Andrey

Matson, Johnny

Mattila, Leena

Matvievski, Vladimir

Mavropoulou, Sophia

Mawle, Elizabeth

Maybery, Murray

Mayes, Linda

Mayes, Susan Dickerson

Mayo, Jessica

Mazefsky, Carla

Mazurek, Micah

Mazur-Kolecka, Bozena

Mazzone, Luigi

McAlonan, G.M.

McCanlies, Erin

McCartney, Patricia

McCleery, Joseph

McConachie, Helen

McCracken, James
McCrary, Stephen

McCrimmon, Adam

McCrory, Eamon

McDonough, Joyce

McDougle, Christopher

McDuffie, Andrea

McEvoy, Linda $\mathrm{K}$

McFadden, Kathryn

McFarland-Whisman, Jennifer

McGonigle-Chalmers, Margaret

McGrath, John

McGrath, Lauren

McGregor, Karla

McGrew, John

McHale-Molenda, Mary

McHugh, Louise

McIntosh, Daniel

McIntosh, David

McLaughlin, Sharon

McLennan, John

McMahon, William

McNamara, Kevin

McNaughton, David

McPartland, James

McPheeters, Melissa

Mechling, Linda

Meerkerk, Gert-Jan

Meiser-Stedman, R.

Menezes, Camila

Merchán-Naranjo, Jessica

Messinger, Daniel

Meyer, Echo

Meyers, Judith

Michaels, Craig

Michaels, Suzanne

Miles, Judith

Miller, Arnold

Miller, Carol

Miller, David

Miller, Judy

Milne, Elizabeth

Milne, Rebecca

Milne, Susan

Miltenberger, Raymond

Mineo Mollica, Beth

Minio-Paluello, Ilaria

Miniscalco, Carmela

Minjarez, Mendy

Minshawi, Noha

Minshew, Nancy

Mire, Sarah

Mirenda, Pat

Misri, Shaila
Mitchell, J.E.

Mitchell, Peter

Molesworth, Catherine

Molloy, Cynthia

Monari Martinez, Elisabetta

Mongillo, Elizabeth

Monk, Christopher

Montes, G.

Montgomery, Janine

Montgomery, Joyce

Moore, James

Mora, Renzo

Moran, Michael

Mori, Kyoko

Morrier, Michael

Morris, Robin

Mosconi, Matthew

Moss, Joanna

Mosse, Emma

Mostofsky, Stewart

Mothander, P.

Mottron, Laurent

Mouridsen, Kim

Mouridsen, Svend Erik

Moy, Sheryl

Mulick, James

Müller, Ralph-Axel

Mulligan, Aisling

Mundy, Peter

Muns, Shawna

Munson, Jeff

Murdock, Linda

Murias, Michael

Murphy, Declan

Murphy, Deirdra

Murray, Donna

Murray, Michael

Musiek, Frank

Muskett, Tom

Myers, Scott

Myles (Smith-Myles), Brenda

Naber, Fabienne

Nadel, Stephen

Nadig, Aparna

Nadon, Geneviève

Nah, Yong-Hwee

Naigles, Letitia

Najjar, F.

Nakahachi, Takayuki

Nakamura, Kazuhiko

Nakano, Tamami

Naoi, Nozomi

Naples, Adam 
Narita, Naoko

Nation, Kate

Natowicz, Marvin

Naylor, Paul

Neece, C.

Negri, Lisa

Nelson, Charles

Nelson, Linda

Nelson, Phillip

Ness, Kirsten

Neumann, Nicola

Neumeyer, Ann

Nevill, Rose

New, J.J.

Newacheck, Paul

Nicolson, Rob

Nippold, Marilyn

Noelle, David

Noens, Ilse

Noland, Julia

Norbury, Courtenay

Nordquist, Vey

Noterdaeme, Michele

Novins, Douglas

Nurnberger, John I.

Nygren, Gudrun

Oberman, Lindsay

O'Callaghan, F.V.

Odom, Samuel

O'Donnell, Brian

Oetting, Janna

Offit, Paul

Ogliari, Anna

O'Hare, Anne

O'Hearn, Kirsten

Oi, Manabu

Olfson, Mark

Olive, Melissa

Oliver, Chris

Ollendick, Thomas

Oller, Kim

Oller, Stephen

Ølund Birkeland, Silje

O'Meara, Grace

Ooi, Yoon Phaik

Oosterling, Iris

O'Reilly, Mark

O'Reilly, Michelle

Orellana, Carmen

Orsmond, Gael

Osada, Hirokazu

Osborne, Lisa

Osei, Albert
Oslejskova, Hana

Osorio, Ivan

Osterling, Julie A

Ostryn, Cheryl

Oswald, Donald

Oti, Rosalind

Ousley, Opal

Overton, Terry

Owley, Thomas

Ozcaliskan, Seyda

Ozdemir, Selda

Ozonoff, Sally

Palcevski, Goran

Pan, C.Y.

Pandolfi, Vincent

Panerai, Simonetta

Paparella, Tanya

Papoudi, Despina

Paradis, Johanne

Pardo, Carlos

Parellada, Mara

Parish-Morris, Julia

Park, J.H.

Parkes, Georgina

Parner, Erik

Parr, Jeremy

Partington, Carrie

Partington, James

Paterson, Sarah

Patterson, Paul

Paul, Rhea

Pauls, David

Pearson, Deborah

Pei, Francesca

Pelangka, Sarah

Pellicano, Elizabeth

Pelphrey, Ph.D, Kevin A.

Pelucchi, Bruna

Pelzel, Kelly

Pennington, Bruce

Pepa, Lauren

Pepler, Debra

Peppe, Susan

Pereira, Tatyana

Perkins, Tom

Perlman, Susan

Pernon, E.

Perrin, Ellen

Perrin, James M.

Persico, Antonio

Peter, Stacy

Peterson, Bradley

Peterson, Candida C.
Pexman, Penny

Pfaltz, Monique

Pfeffer, Rebecca

Pfeiffer, Beth

Phalen, James

Phelan, Mary

Phetrasuwan, S.

Philofsky, Amy

Picard, Rosalind

Pickles, Andrew

Pierce, Karen

Pinborough-Zimmerman, Judith

Pine, Daniel

Pineda, Jaime

Pinkham, Amy E.

Pinto-Martin, J.A.

Pisula, Ewa

Pitskel, Naomi

Pittman, Kenneth

Piven, Joseph

Plaisted, Kate

Planche, Pascale

Plantin, L.

Pletnikov, Mikhail

Ploog, Bertram

Poehlmann, J.

Poljac, Edita

Poncin, Yann

Poon, Kenneth

Porges, Stephen

Porter, Melanie

Posey, David

Posserud, Maj-Britt

Poustka, Fritz

Powers, Kristen

Powers, Michael D.

Prandota, Joseph

Prasad, Suyash

Pratt, Cathy

Preissler, Melissa

Prelock, Patricia

Press, Clare

Preston, Jonathan

Price, Johanna

Price, Kelly

Price, Lawrence

Pring, Linda

Probst, Paul

Provost, Beth

Pry, René

Puleo, Connor

Pumain, Rene

Quintin, Eve-Marie 
Quirmbach, Linda

Rabadi, Nazar

Rabins, Peter

Rada, Robert

Rahbar, Mohammad

Raj, Vimal

Raja, Michele

Rajendran, Gnanathusharan

Ramsay, Gordon

Rao, Patricia

Rapoport, Judith

Rasouli, Soheyla

Ratzon, N.Z.

Rauch, A.

Rausch, Jeffrey L.

Raymaekers, Ruth

Rayner, C.S.

Reaven, Judy

Reed, Catherine

Reed, Phil

Reed, Vicki

Reese, R. Matthew

Reichle, Joe E.

Reichow, Brian

Reisinger, Lisa

Reiss, David

Remington, Anna

Rensink, Ron

Rexroth, Daniel

Reznick, J. Steven

Rhodes, Sinead

Ribeiro do Valle, Sandra Lie

Riby, Deborah

Riccio, Cynthia

Rice, Catherine

Rice, Robert

Richdale, Amanda

Richler, Jennifer

Rieffe, Carolien

Rimmer, J.H.

Rinehart, Nicole J.

Ring, Alexander

Ringdahl, Joel

Risi, Susan

Ritvo, Riva

Rivkin, Judith

Robb, Michael

Roberts, Jane

Roberts, Joanne

Roberts, Timothy

Robins, Diana

Robinson, Sally

Robson, Danielle
Rockcastle, Margaret

Roesser, Jessica

Rogers, Kimberley

Rogers, Sally J.

Rojahn, Johannes

Rollins, Pamela

Roll-Pettersson, Lise

Romanczyk, Raymond

Rommelse, Nanda

Romski, Mary Ann

Ronald, Angelica

Roos, Elizabeth

Ropar, Danielle

Rosbergen, Alke

Rosenberg, David

Ross, Randy

Rossignol, Daniel

Rowlandson, Piers

Rozga, Agata

Rubin, David

Rubin, Julie

Ruble, Lisa

Rudolph, K.

Ruiz, Doraelia

Rule, Nicholas

Russell, Ailse

Russell, James

Russell-Smith, Suzanna

Russo-Ponsaran, N.

Rust, Jonathan

Ruta, Liliana

Rutgers, Anna H.

Rutherford, M.D.

Rutter, Michael

Ryan, Christian

Sachse, Michael

Saeed, Tahir

Saemundsen, Evald

Sakkalou, Elena

Saldana, David

Sale, Liz

Salimpoor, Valorie

Salvano-Pardieu, Veronique

Samadi, Sayyed Ali

Samson, Andrea

Sandfeld Nielsen, Lisbeth

Sandford, Cheryl

Sankari, Ziad

Sansone, Stephanie

Sansosti, Frank

Santangelo, Tanya

Sarafidou, Maria

Sasson, Noah
Saulnier, Celine

Savage, Sue

Scahill, Lawrence D.

Scambler, D.J.

Scassellati, Brian

Schaaf, Roseann

Schacht, Patricia

Schachter, Steven

Schaefer, Mark

Schall, Carol

Schanding, George

Scharf, Jeremiah

Scheeren, Anke

Schendel, D.E.

Scherer, Stephen

Scherf, Suzanne

Schieve, L.A

Schilbach, Leonhard

Schlaug, Gottfried

Schmidt, Gwenda

Schmidt, Rebecca

Schmitz, Christina

Schoen, Elizabeth

Schonherz-Pine, Yael

Schrandt, Jessica

Schreibman, Laura

Schultz, Robert

Schultz, Tia

Schumann, Cynthia

Schutz-Bosbach, Simone

Schwartz, Caroline

Schweitzer, Julie

Scott, Eric

Scott-Van Zeeland, Ashley

Scullard, P.

Seif Eldin, Amira

Seiverling, Laura

Self, Trisha

Seltzer, Marsha

Semrud-Clikeman, Margaret

Senecky, Yehuda

Senel, Hatice

Senju, Atsushi

Seung, Hye-Kyeung

Sevick, Rose

Shafritz, Keith

Shamay-Tsoory, Simone

Shams, Ladan

Shane, Howard

Shapiro, Eugene

Shattuck, Paul

Shaw, Tracey

Shea, Victoria 
Shechner, T.

Shelton, Janie

Shen, Yipling

Sheppard, Elizabeth

Sheridan, B.A.

Sherry, Lori

Sheth, Bhavin

Shic, Fred

Shimada, S.

Shon, Seoyoung

Shriberg, Lawrence

Shtayermman, Oren

Shu, Bih-Ching

Shulman, Cory

Shumway, Stacy

Shurtleff, T.L.

Siaperas, Panagiotis

Sidhu, Reet

Siegel, Bryna

Siegel, Matthew

Sigafoos, Jeff

Sikich, LinMarie

Sikora, Darryn

Siller, Michael

Silton, Nava

Silverman, Jeremy

Silverman, Laura

Siman Tov, Ayelet

Simmons, Alan

Simmons, David

Simon, Eileen

Simon, Tony

Simonoff, Emily

Simpson, Richard L.

Singer, Alison

Singh, Nirbhay

Sinzig, Judith

Siqueira, José Tadeu

Skelton, J.A.

Skuse, David

Slaughter, Virginia

Slayter, Elspeth

Slonims, Vicky

Small, Joyce

Smerbeck, Audrey

Smith, Brenda

Smith, Cary Stacy

Smith, Gwenn

Smith, Isabel

Smith, Leann

Smith, Shelley

Smith, Stephen

Smith, Tristram
Smith, Veronica

Smithson, Pontea

Snell, Martha E.

Snider, Laurie

Snowling, Maggie

So, Pety

Sofronoff, Kate

Sokhadze, Estate

Solomon, Marjorie

Somogyi, Eszter

South, Mikle

Sowden, Hannah

Spector, Janet

Spek, Antoinette

Spence, Sarah

Sperry, Laurie

Spezio, Michael

Spiker, Donna

Spinrad, T.

Spratt, Eve

Spreckley, Michele

Spreng, Nathan

Squires, Nancy

Stanfield, Andrew

Stanley, Gillian

Stanley, Maria

Starr, Elizabeth

State, Matthew

Staufenberg, Ekkehart

Steele, Shelly

Steiner, Amanda

Stel, Marielle

Stenberg, Georg

Stephens, Carolyn

Sterkenburg, Paula

Sterling, Lindsey

Stevens, Hanna

Stevenson, Bruce

Stewart, Ian

Stewart, Mary

Stichter, Janine

Stieglitz Ham, Heidi

Stigler, Kimberly

Stoddard, Joel

Stoddart, Kevin

Stokes, Mark

Stone, Wendy

Stoneman, Zolinda

Storz, Michael

Stover, Carla

Strahler, Jana

Strauss, Mark

Stribling, Penny
Stronach, Sheri

Strough, JoNell

Stubbe, Dorothy

Studenka, B.E.

Sturmey, Peter

Sukhodolsky, Denis

Sullivan, Katherine

Sulzer-Azaroff, Beth

Sumiyoshi, Chika

Summers, Jane

Sumner, Jennifer

Sunaert, Stefan

Suri, Neeti

Susman, E.

Swanson, Jim

Sweeney, John

Sweeten, Thayne

Sweller, Naomi

Swettenham, John

Swiezy, N.

Sylvester, Christin

Symon, Jennifer

Symons, Frank

Szatmari, Peter

Szymanski, Christen

Tadic, Valerie

Taffoni, Fabrizio

Tager-Flusberg, Helen

Takarae, Yukari

Takeuchi, Naoyuki

Tanaka, Hiroki

Tanaka, James W.

Tanaka, Yuko

Tang, Kitty

Tanguey, Peter

Tantam, Digby

Tarnai, Balazs

Taurines, R.

Tavares, Wendy

Taylor, Julie

Taylor, Lauren

Teder-Salejarvi, Wolfgang

Tedesco, Daniel

Teitelbaum, Philip

Teixeira, M.C.

Tek, Saime

Tekin-Iftar, Elif

Tepest, Ralf

Thibert, Ron

Thiemann, Kathy

Thierfeld Brown, Jane

Thioux, Marc

Thomas, Michael 
Thompson, Jim

Thompson, Lynda

Thompson, Richard

Thompson, Robyn

Thony, Beat

Thurm, Audrey

Thyer, B.A.

Timmerman, Lorna

Tissot, C.

Todd, Gabrielle

Tolosa, Amparo

Tomasello, Michael

Tomchek, Scott

Tomonaga, M.

Tonge, Bruce

Tordjman, Sylvie

Toriello, Helga

Toth, Karen

Totsika, Vasiliki

Towbin, Kenneth

Townsend, Jeanne

Tracy, Jessica

Travers, Jason

Treffert, Darold A.

Trepagnier, Cheryl

Tsai, Luke

Tsakanikos, Elias

Tsang, Sandra

Tsatsanis, Katherine

Tse, Jeanie

Tsiouri, Ioanna

Tsuchiya, Kenji

Tully, Tim

Turk, Jeremy

Turkstra, Lyn

Turner-Brown, Lauren

Twoy, R.

Uchiyama, Tokio

Uddin, Lucina

Uhlhaas, Peter

Underwood, Lee

Underwood, Lisa

Uono, Shota

Vaccarino, Flora

Vaidya, Chandan

Valdovinos, Maria

Valenti, Marco

Valicenti-McDermott, Maria

Valla, J.M.

Vallino, Linda

van Bakel, Marit

van Berckelaer-Onnes, Ina A.

van Bergeijk, Ernst van Bourgondien, Mary E.

van Bysterveldt, Anne

van Cleave, Jeanne

van de Water, Judy

van den Akker, A.L.

van den Eijnden, Regina

van der Gaag, Rutger

van Engeland, Herman

van Eylen, Lien

van Huffel, Sabine

van Laarhoven, Toni

van Lancker Sidtis, Diana

van Roekel, Eeske

van Rooij, Antonius

Vandenbroucke, Myriam

Vander Wyk, Brent

Vanvuchelen, Marleen

Vasa, Roma

Vasconcellos, Mariliz

Vaughan van Hecke, Amy

Veenstra-Vanderweele, Jeremy

Ventola, Pamela

Verhoeven, Lisette

Vernon, David

Vernon, Ty

Vervloed, Mathijs

Vignoli, Aglaia

Villa, Susanna

Virues-Ortega, Javier

Vismara, Laura

Visootsak, J.

Vital, Pedro

Vitulano, Lawrence

Vivanti, Giacomo

Vohra, Sunita

Voigt, Robert

Volden, Joanne

Vriend, Jennifer

Wachtel, Karen

Wagner, Shannon

Wai Wan, Ming

Waiter, G.D.

Wakabayashi, Akio

Wakeford, Linn

Waldman, H.B.

Wallace, Greg

Wallace, Simon

Walsh, Christopher

Wang, Hui-Ting

Ward-King, Jessica

Waring, Rosemary

Warren, Zachary

Wasilewska, Jolanta
Wass, Sam

Watanabe, Yoriko

Waterhouse, Lynn

Watling, Renee

Watson, Duane

Watson, Linda

Watt, Nola

Weaver, Priscilla

Webb, Sara

Webber, Emily

Weber, Wendy

Webster-Stratton, Carolyn

Weeks, Murray

Weidenheim, Karen

Weidle, Bernhard

Weisblatt, Emma

Weiser, Mark

Weiss, Deborah

Welsh, Timothy

Welterlin, Aurelie

Wentz, Elisabet

West, Constance E.

Westphal, Alexander

Westphal, Rebecca

Wetherby, Amy

Whalen, Christina

Wheelwright, Sally

White, Catriona

White, Sarah

White, Susan

Whitehouse, Andrew

Wiest, Michelle

Wiggins, Lisa

Wilcox, Jeanne

Wilkinson, Krista M.

Williams, David

Williams, Dean

Williams, Diane

Williams, Joanna

Williams, Justin

Williams, Katrina

Williams, Marian

Williams, Susan

Wilnarger, Julia

Wilson, Ellie

Wimmer, M.C.

Wimpory, Dawn

Wimsatt, Amber

Windham, Gayle

Wing, Lorna

Winskel, $\mathrm{H}$.

Wodka, Ericka

Wolf, Jonathan 
Wolf, Julie

Wolff, Jason

Wolraich, Mark

Wolters, Pamela

Wong, Dana

Wong, Dean

Wong, Kwan

Wong, Nina

Wong, Virginia

Wood, Jeffrey

Wood, Larry

Woodard, Cooper

Woodbury-Smith, Marc

Woods, James

Woods, Juliann
Woodward, Jason

Woolston, Joseph

Wozniak, Janet

Wright, Barry

Yama, Brie

Yanarda $\breve{g}$, Mehmet

Yang, M.

Yang, Sijun

Yates, Tuppett M.

Yazgan, Yanki

Yeargin-Allsopp, Marshalyn

Yerys, Benjamin

Yirmiya, Nurit

Yoder, Paul J.

Yoon, En-Ho
Young, Edna

Young, Gregory

Yuill, Nicola

Zachor, Ditza

Zajac, David

Zalla, Tiziana

Zampini, Massimiliano

Zanolli, Kathleen

Zermatten, Ariane

Zhang, Xuyang

Zilbovicius, Monica

Zimmerman, Andrew

Zinke, Katharina

Zucker, Kenneth

Zwaigenbaum, Lonnie 